\title{
REPLICATING HUMAN INTERACTION IN BRAESS PARADOX
}

\author{
Arianna Dal Forno \\ Department of Economics \\ "Cognetti de Martiis" \\ University of Torino \\ lungo Dora Siena 100A \\ I-10153 Torino, ITALY
}

\author{
Ugo Merlone \\ Department of Psychology \\ University of Torino \\ via Verdi 10 \\ I-10124 Torino, ITALY
}

\begin{abstract}
The Braess Paradox shows how adding a new road to a traffic network may actually increase the total travel time. It has recently found new interest in research. Researchers conducted new experiments with human participants in order to observe the outcomes with an increasing number of people, with private or public monitoring. A small number of papers were devoted to the observation of different behaviors, and intuitively suggested some theoretical hypotheses about the heterogeneity of the participants. Analyzing the data gathered from the observation of an experiment with human participants, and coding artificial behaviors emerged by mean of Grounded Theory, we used ABM simulations to confirm or disprove possible behaviors and composition of the population that was so far suggested only theoretically.
\end{abstract}

\section{INTRODUCTION}

Social dilemmas arise from collective actions and are situations in which each individual has a clear and unambiguous incentive to make a choice that provides a poorer outcome for all when it is made by all individuals than the outcome they would have received when none of them had made the choice (Dawes and Messick 2000, p. 111). Social dilemmas have been studied in several disciplines: economics, psychology, sociology among the others.

In some cases social dilemmas can be considered as multiple choice problems. For example, in the well known prisoner's dilemma the two choices are cooperating or defecting. This dilemma can be generalized as an $N$-person dilemma, see for example Hauert and Szabo (2003). The dynamics of agent populations interacting in prisoner's dilemma have been analyzed extensively both in two-person version (Axelrod 1984) and N-person version (Merlone, Sandbank, and Szidarovszky 2012; Merlone, Sandbank, and Szidarovszky 2013). When the number of choices increases the situation is more complex. While the dynamics of binary choices has been extensively studied theoretically (Bischi and Merlone 2009; Bischi, Gardini, and Merlone 2009a; Bischi, Gardini, and Merlone 2009b; Gardini, Merlone, and Tramontana 2011) the contributions considering ternary choices are relatively recent and more limited in number (Dal Forno, Gardini, and Merlone 2012; Dal Forno and Merlone 2013). With several choices the theoretical analysis becomes more difficult and, when considering heterogeneous behaviors, simulation may provide interesting insights (Santos, Pacheco, and Lenaerts 2006).

An interesting social dilemma involving three choices has been proposed in Braess (1968). This dilemma, known as the Braess paradox, has captured the interest of several scholars and practitioners not only in transportation science (Smith 1979, Steinberg and Zangwill 1983) but also in computer science (Boyce, Mahmassani, and Nagurney 2005) and economics (Rapoport et al. 2008).

The Braess paradox can be illustrated by the following example as depicted in Figure 1. Assume there is a unitary mass of commuters from Start (S) to End (E), and there are two roads, one passing through Left (L) and the other through Right (R). Time spent along segments S-R and L-E does not depend on 


\section{Dal Forno and Merlone}

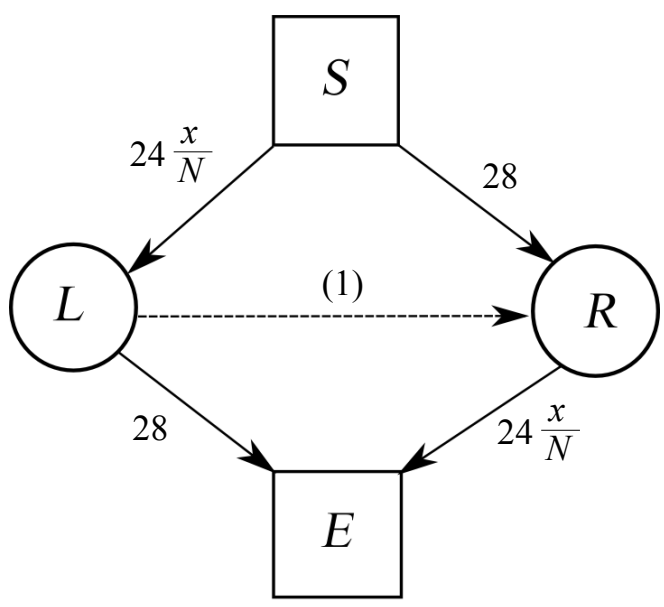

Figure 1: Braess paradox. Adding the dashed edge to the network the performance worsen.

traffic and it costs 28 minutes; on the contrary, time spent along segments S-L and R-E is proportional to the number of commuters and it costs $24 x / N$ minutes, where $x \in\{1, \ldots, N\}$ is the number of commuters using the segment. There is a Nash equilibrium with commuters splitting at $\mathrm{S}$, so that both roads cost in the whole 40 minutes. Now we assume that a very fast road is built connecting $\mathrm{L}$ to $\mathrm{R}$ that costs 1 minute. There is a new Nash equilibrium where the entire population chooses path S-L-R-E with 49 minutes total cost. Therefore, adding a link to a network with linear costs depending on congestion, when each user independently seeks her best possible route, the total travel time may increase.

In Dal Forno and Merlone (2013) the Braess paradox was studied considering impulsive agents. With impulsive agents a fraction of agents immediately switches strategy even when the difference between costs is extremely small (for a discussion about impulsive agents in binary choices see Bischi, Gardini, and Merlone (2009a)). The fraction of agents switching to a lower cost road is modeled by parameters $\delta_{L}, \delta_{L R}$ and $\delta_{R}$ all in $[0,1]$. As this behavior seems quite extreme, in this paper we consider also agents where the switching rate is proportional to the difference of payoff (we are grateful to Amnon Rapoport for suggesting us such a behavior). Finally, we analyze the human participants' behavior using Grounded Theory (Glaser and Strauss 1967, Strauss and Corbin 1998). From our analysis two kinds of behaviors emerged, impulsive and radical. Impulsive agents' behavior was quite similar to the one considered in Dal Forno and Merlone (2013). Radical agents on the contrary kept following the same path even when it provided a higher cost; a similar behavior was observed also in Rapoport et al. (2009). In Section 2 we illustrate the experimental design. The full analysis of agents' behavior and the computer code we used to model them is reported in Section 3. The results obtained with simulations and the discussion are in Section 4. Finally, the last section is devoted to conclusions and further research.

\section{THE EXPERIMENTAL DESIGN}

Several experiments with human participants have been conducted on Braess Paradox. Some gave support to the claim that as the number of interacting agents grows, altruism, reciprocity, punishment, and tacit coordination-that are quite effective in sustaining cooperation in some non-cooperative games with deficient equilibria when $\mathrm{n}=2$-quickly lose their effectiveness and potency when $\mathrm{n}$ increases (Rapoport, Mak, and Zwick 2006). Others investigated the effect of private monitoring, that is, at each turn individuals were informed only of their performance and not of the whole network performance as in public monitoring (Morgan, Orzen, and Sefton 2009). Yet, no patterns of route choice that increase efficiency were observed (Gisches and Rapoport 2012).

Our experiment was with public monitoring and aimed to collect different behaviors observed in the literature (Rapoport et al. 2009) to properly model behaviors in artificial populations. The main contribution 


\section{Dal Forno and Merlone}

of this paper is to build a bridge between experimental and simulation methodology in order to provide a tool to explore and analyze different scenarios when the population in not homogeneous.

The experiment was run in one day and it was divided in two phases. In the first phase, one of the authors gave a detailed description of the traffic game with only two roads to the participants. Numerical examples were provided to improve the understanding of the game. After being so instructed, the participants played the game for ten rounds. Then, in the second phase, the participants received the detailed description of the same game but with the link that connects the left road to the right one. Again, numerical examples were provided, after which the participants played the game for eigth rounds.

Since our focus in the experiment was behavioral modeling, the detailed explanation of participants' choices was crucial. Therefore, during each round we asked the participants to explain the reasons for their choices. These additional data provided by the participants allows us to check coherence and to collect both quantitative and qualitative data. While some patterns of behavior may be common to different subjects, the motivations may be different. This makes possible the partitioning of different behaviors in homogeneous classes (obviously, other approaches such as cluster analysis are possible).

Our next step, was the codification of the behavioral classes and so to characterize different types of agents according to the Grounded Theory principles (for further details, see Dal Forno and Merlone (2012)). Motivating subjects in experiments is a well-known problem. The students were encouraged to participate to the experiment receiving up to two extra credits additional to their final mark, dependently on the performance in the whole game. The data obtained in the experiment were subsequently used to perform some agent-based model simulations.

We are aware that the number of participants involved is too small to provide a solid empirical basis for generalizations. Yet, the behaviors we observe are important since they both provide further justification of individual heterogeneity and may suggest alternative models of bounded rationality - which are all important aspects when approaching the modeling of artificial agents behaviors.

As regards symmetric equilibria, the correspondent one-shot game with only two roads has a unique Nash equilibrium in mixed strategies, that is also Pareto optimum. At the equilibrium every player chooses the left road with probability $1 / 2$ and with probability $1 / 2$ the right road. The game with the link has still the same Nash equilibrium for $d>16$ where $d$ is the travel time for the new link. For $4<d<16$ the road SLRE strictly dominates every other strategy and the unique Nash equilibrium in pure strategies (that is not Pareto efficient) consists of every commuter using the road SLRE. A Pareto optimal coordination equilibrium is possible with players using again the left road with probability $1 / 2$ and the right road with probability $1 / 2$. Of course, there is a strong incentive to deviate and this gives the cooperative equilibrium a very low probability of being played.

Here we report the data from undergraduates who were in the Math class at the Business Administration School of the University of Torino. The participants were 20 females and 17 males with average age 21.68 and standard deviation 4.55. We ran the first experiment with only two roads, and then the second experiment, with the same 37 participants with the link added. The first experiment consisted of 10 rounds and the second consisted of 8 rounds. At the beginning of each round, every student was asked to choose a road, in order to minimize his/her individual cost, and to provide a short motivation for their decision.

Each participant was allowed to use any computational tool to make his/her decision but no communication was allowed during each round. After each round a public updated table was given with the number of commuters present in any road, and individual costs.

In Table 1, we report the result of the experiment. For each round we provided the number of commuters in any road of the game. Figure 2 depicts the dynamics we observed in the experiment. Oscillations start at turn 4 and continue with different amplitude. They occur as the relative majority switches between the two available roads; these cycles are coherent with the theoretical findings about impulsive behavior provided in Bischi, Gardini, and Merlone (2009a). When at turn 11 the link becomes available the two existing roads are progressively abandoned as the number of agents using the new link increases. It is worth to observe that some oscillations with the agents using the initial roads remain. 
Table 1: Experiment results. For each round, the number of commuters in any particular road is provided. The game with two roads was played for 10 rounds, while the game with three roads for 8 rounds.

\begin{tabular}{l|rrrrrrrrrrrrrrrrrr} 
Round & 1 & 2 & 3 & 4 & 5 & 6 & 7 & 8 & 9 & 10 & 11 & 12 & 13 & 14 & 15 & 16 & 17 & 18 \\
\hline S-L-E & 24 & 21 & 21 & 17 & 19 & 16 & 23 & 15 & 13 & 22 & 13 & 7 & 4 & 5 & 1 & 1 & 0 & 0 \\
S-L-R-E & & & & & & & & & & & 14 & 22 & 28 & 30 & 34 & 34 & 36 & 34 \\
S-R-E & 13 & 16 & 16 & 20 & 18 & 21 & 14 & 22 & 24 & 15 & 10 & 8 & 5 & 2 & 2 & 2 & 1 & 3
\end{tabular}

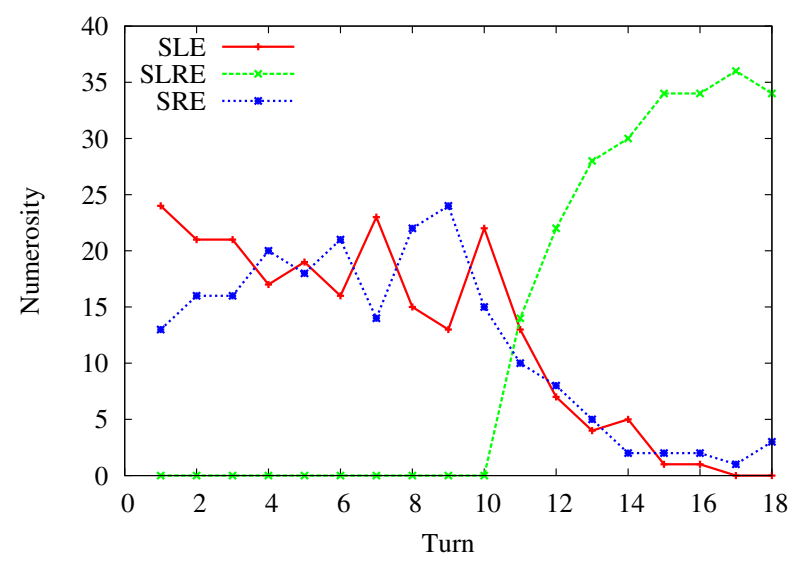

Figure 2: Human population consisting of 37 participants. The horizontal axis represents turns and the vertical one the number of participants distributed in each available road at that turn; the different choices are represented with different colors. At the first turn 24 people played SLE and 13 played SRE. The new link was added at turn 11. Lines connecting dots are for illustrative purpose only.

\section{GROUNDED THEORY AND AGENTS' BEHAVIOR CODING}

The Grounded Theory approach is a qualitative research method proposed by Glaser and Strauss (1967) and later developed by Strauss and Corbin (1998) to analyze and interpret data. The basic idea is to read a textual database several times and find the interrelations between some aspects that emerge during the process. Starting with a research, where the goal is to find what is happening or how the players are managing their roles, the researcher writes down the observed behaviors. Then, by the analysis and the constant comparison of the data, some of the observed phenomena are labeled and become concepts. As concepts begin to accumulate, they are grouped under more explanatory terms named categories. Through this process, as a theory emerges it is continuously compared to the data. The process continues in order to saturate all the categories, that is, when no new information seems to emerge by coding, and we have achieved theoretical saturation, according to the terminology of Strauss and Corbin (1998). By axial coding, categories are related to their subcategories according to their properties and dimensions. Furthermore, the theory is integrated and refined by selective coding. At this point a theory grounded on the data has emerged. Finally, the theory is tested and related to other existing theories.

Recently, some applications of Grounded Theory can be found in other fields besides sociology, as to information systems and to knowledge management. In particular, an example of how Grounded Theory can be used to model participants' behavior in experiments and code it in agent based simulations is given in Dal Forno and Merlone (2012).

Considering the interaction we studied in this paper, the full analysis of agents' behavior is reported in Dal Forno, Giorgino, and Merlone (2013). Here, for the sake of brevity, we only report the coding of the three classes of behavior which emerged. 


\section{Dal Forno and Merlone}

\subsection{Impulsive Agents}

This class of agents is the same as the one considered in Dal Forno and Merlone (2013). When the previous turn route travel time was not the shortest one, a fraction of the agents who followed this route switches to the shortest one. The switching rates are modeled by the parameters $\delta_{\mathrm{SLE}}, \delta_{\mathrm{SLRE}}$, and $\delta_{\mathrm{SRE}}$, depending on which route the agents are switching to. Here we show a fragment of the code that implements this behavior. The displayed section refers to an agent who chose $S L E$; the sections relative to the other choices are similar and therefore not reported.

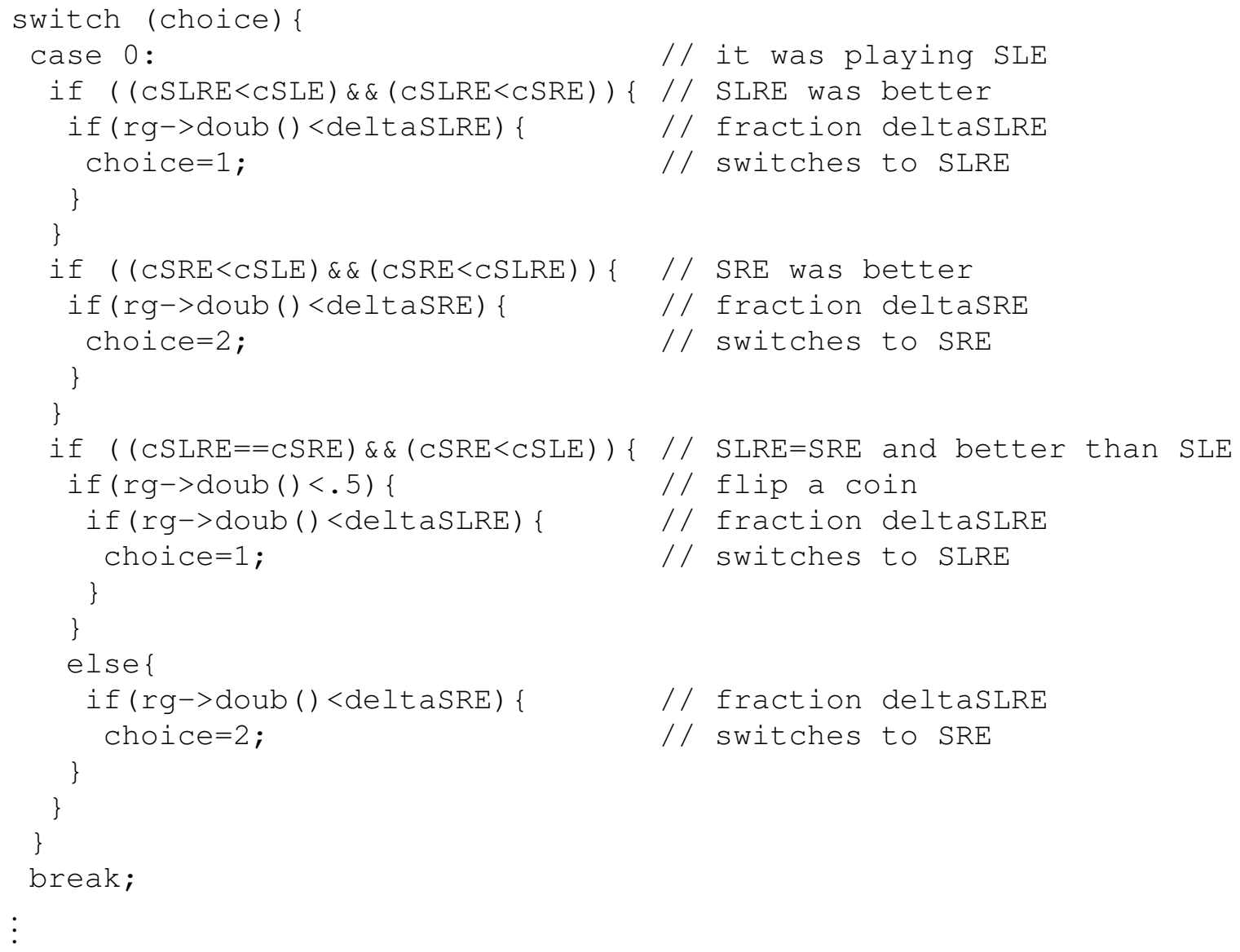

\subsection{Proportional Agents}

This class of agents is similar to the previous one but the switching rates are proportional to the difference in travel times among the routes (we are gratefult to Amnon Rapoport who suggested us to consider this class). Therefore the switching rates are respectively $\delta_{\mathrm{SLRE}}\left(c_{\mathrm{SLE}}-c_{\mathrm{SLRE}}\right) / K, \delta_{\mathrm{SRE}}\left(c_{\mathrm{SLE}}-c_{\mathrm{SRE}}\right) / K$, $\delta_{\mathrm{SLE}}\left(c_{\mathrm{SLRE}}-c_{\mathrm{SLE}}\right) / K, \delta_{\mathrm{SRE}}\left(c_{\mathrm{SLRE}}-c_{\mathrm{SRE}}\right) / K, \delta_{\mathrm{SLE}}\left(c_{\mathrm{SRE}}-c_{\mathrm{SLE}}\right) / K, \delta_{\mathrm{SLRE}}\left(c_{\mathrm{SRE}}-c_{\mathrm{SLRE}}\right) / K$ and depend on the route they are switching to and the route costs in the previous iteration; $K$ is a normalization factor. Also for this class we report the case in which the agent selected route $S L E$ in the previous iteration.

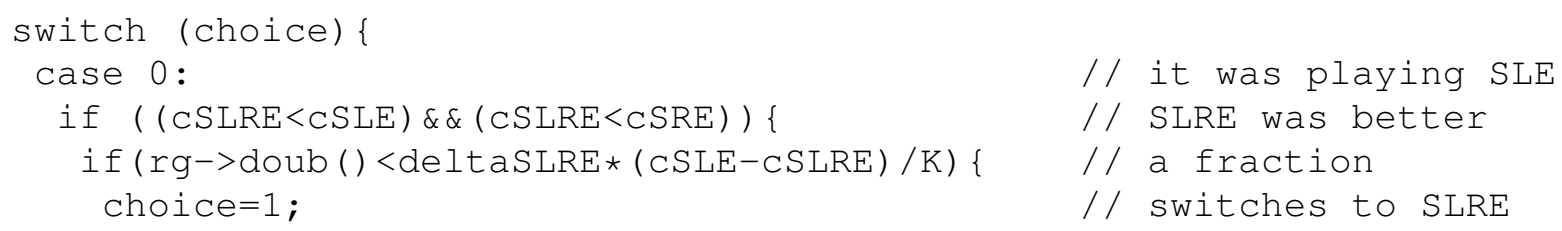




\section{Dal Forno and Merlone}

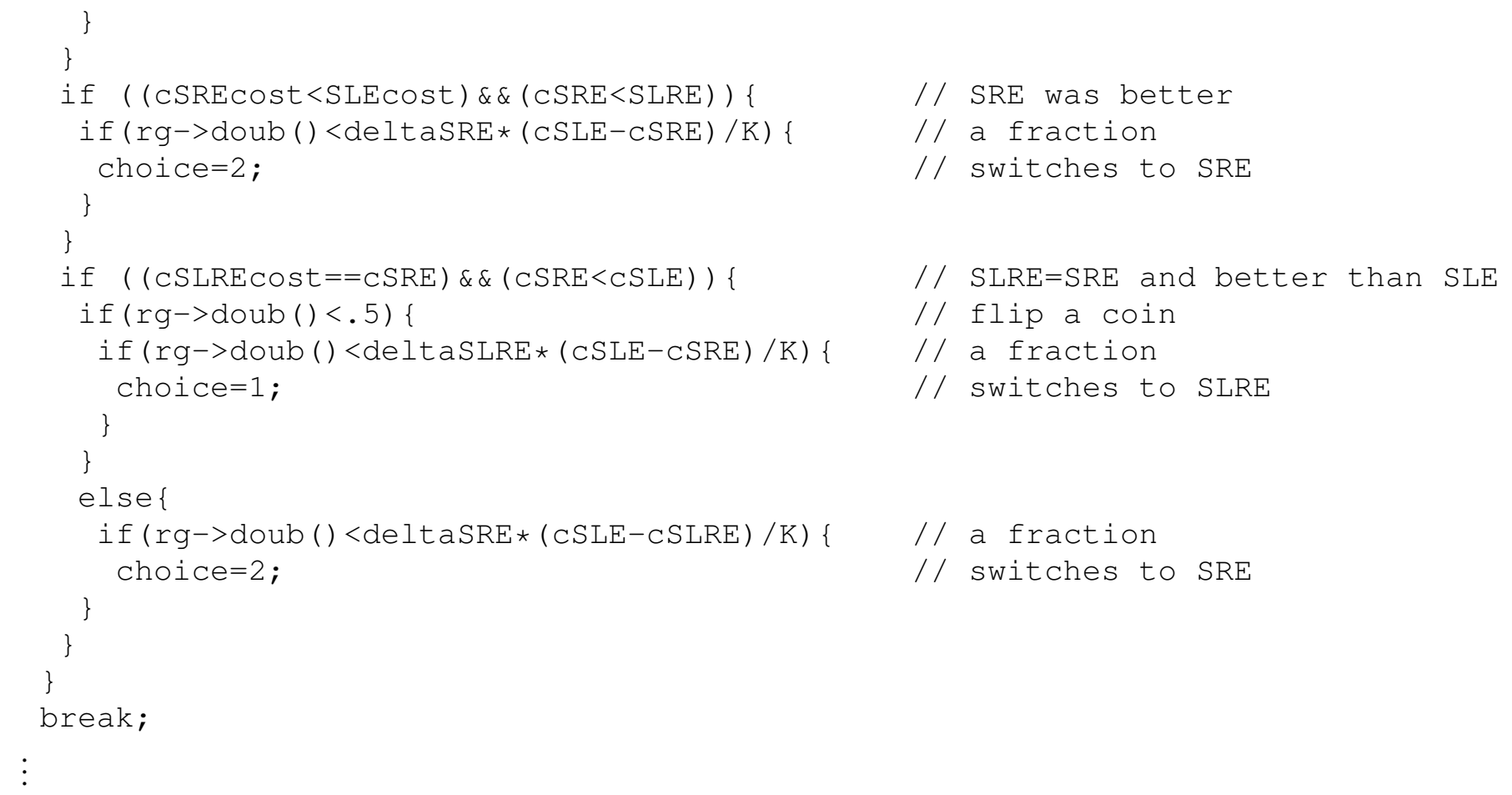

\subsection{Radical Agents}

These agents randomly either choose the route according to the one they have been using most frequently or behave as impulsive agents. Again for the impulsive behavior code we report only the section relative to an agent who chose $S L E$.

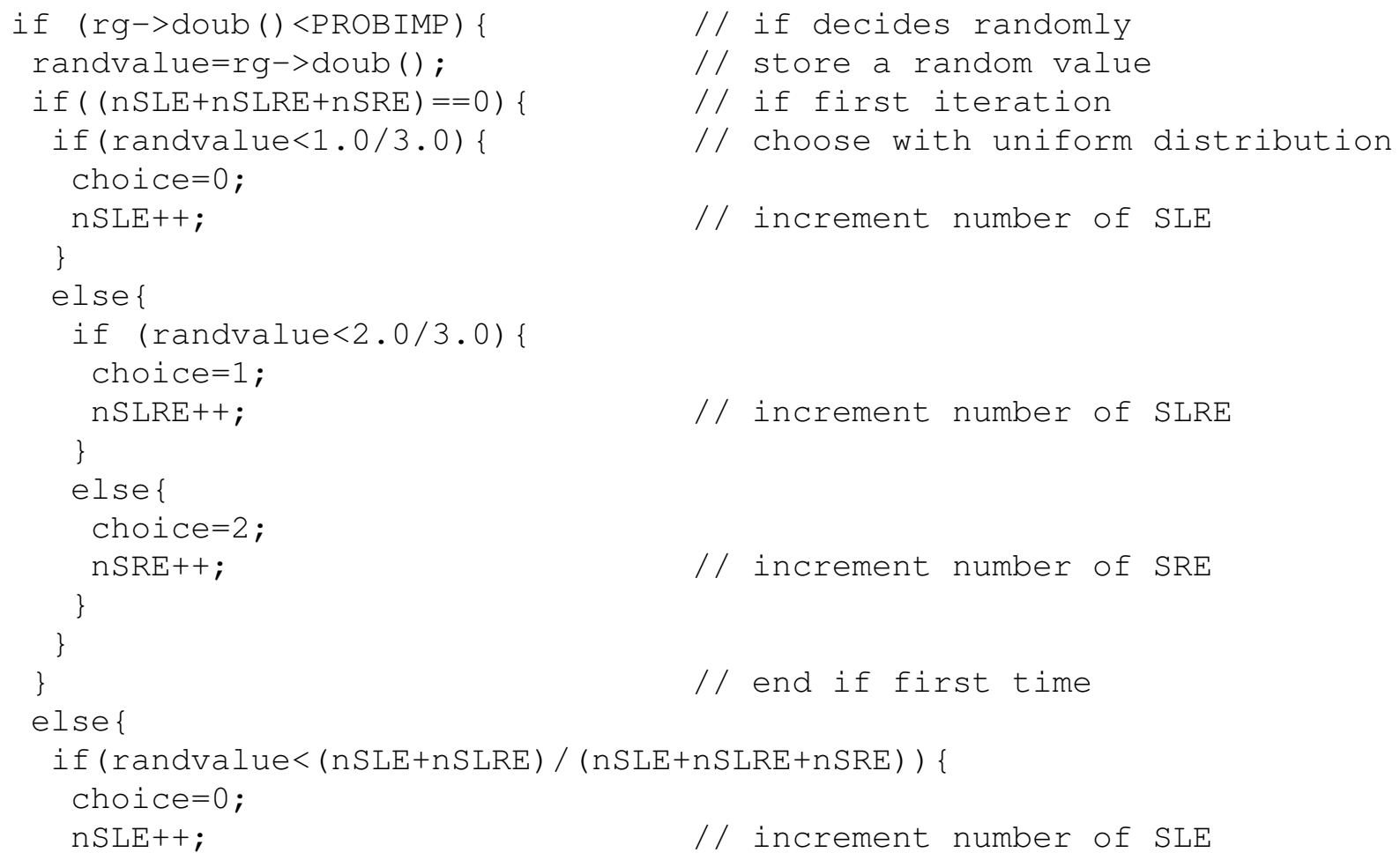




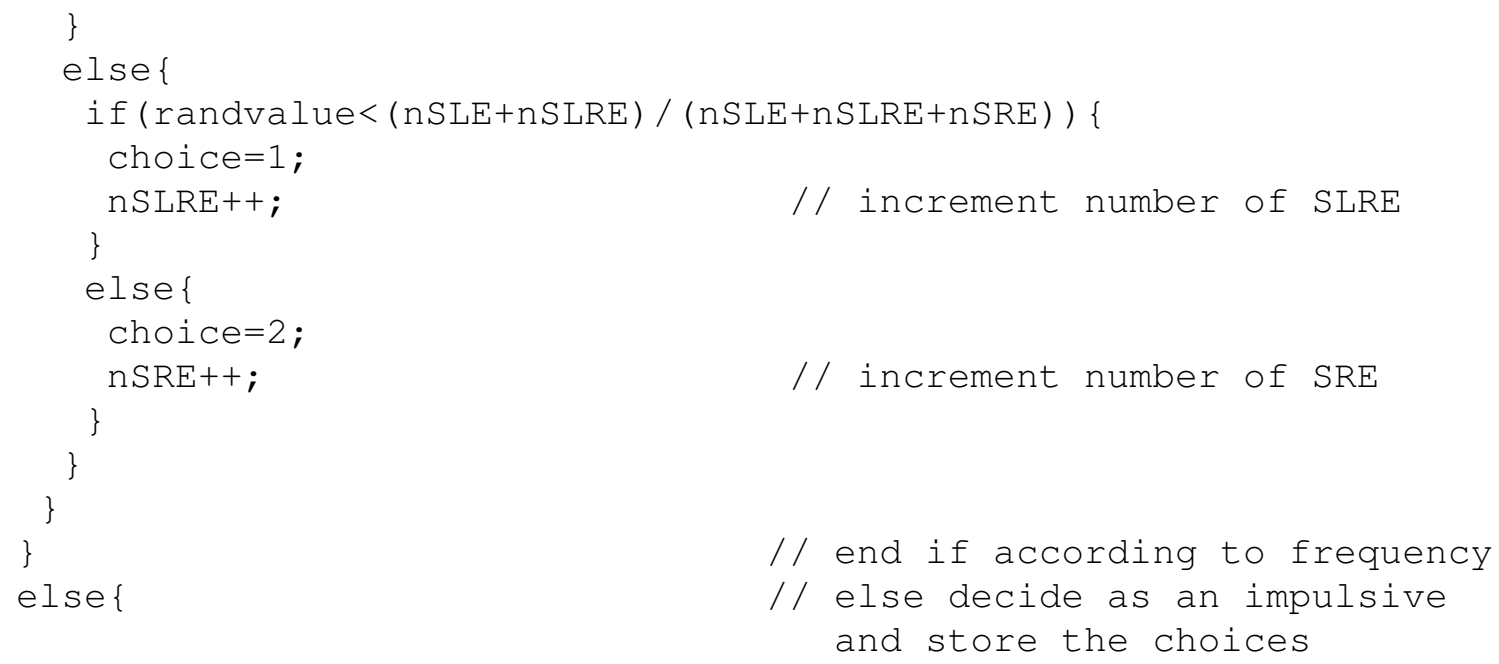

\section{SIMULATION RESULTS AND GRAPHICAL COMPARISON}

In this section we present and compare the results for the different populations. Specifically, we consider homogeneous impulsive, homogeneous proportional, homogeneous radical and heterogeneous populations. The artificial population dynamics are compared to the dynamics we observed in the human participants experiments. We will use graphical analysis as our results are based on a unique human participants experiment. According to Janert (2010) "Graphical analysis is an investigation of data using graphical methods. The purpose is the discovery of new information about the underlying data set". Rather, according to the same Author "statistical analysis always makes some (silent) assumptions about the data that may not be fulfilled in practice. These challenges are well-known in the statistical community", in fact Loftus (1993) provides a seminal contribution on the use of graphical analysis in statistics.

In our case considering some summary statistics of several simulation repetitions would be pointless. On the contrary, we provide the different populations dynamics obtained considering the same random generator seed and varying the population composition. It must be observed that, on changing the random seed, the qualitative behavior of the dynamics keeps its main features when the population composition is fixed. Furthermore, for each different artificial population we performed 1000 repetitions varying the random seed. This analysis allows us to see how the different population dynamics maintains its peculiarity. Every simulation consists of 37 artificial agents, the same number of the participants to the experiment, and the initial choices distribution is the same as the human participants population, i.e., 24 playing SLE and 13 playing SRE. Also, for all the simulations the new link is added at turn 11. This way it is possible to compare the evolution of artificial populations with different behavior compositions. With the homogeneous impulsive population dynamics as reported in Figure 3, as long as only two roads are available the dynamics shows some cycles. Then, as the new link is added at turn 11, all the agents progressively switch to the new route and their travel time increases. With homogeneous proportional population (Figure 4) when only two routes are available we almost have no oscillation and, similarly to the precedent case, the agents switch to the new route when the new link is added; yet, in this case, the fraction of agents switching to the new road depends on the cost difference among roads. Therefore, in equilibrium not all of the agents will be using the new link. With homogeneous radical population (Figure 5) we have initial oscillations and, when the new link is added, the majority of agents follows the new link. In this case, when considering the average the dynamics is quite similar to the one of impulsive agents. In fact, from the computer code it is quite evident that radical agents tend to play the equilibrium in dominant strategies.

For the artificial populations the initial number of agents playing SLE and SLR was respectively 24 and 13. Although this is sufficient to replicate our findings with homogeneous populations, when considering 


\section{Dal Forno and Merlone}

a heterogeneous population the proportion of agents together with their initial choices is necessary. This distribution is reported in Table 2 and takes into account the initial choices and number of radical agents of the human participant experiment.

Table 2: Initial choice distribution for the heterogeneous population.

\begin{tabular}{l|cc} 
& SLE & SRE \\
\hline impulsive & 14 & 4 \\
proportional & 10 & 7 \\
radical & 0 & 2 \\
\hline TOTAL & 24 & 13
\end{tabular}

When considering an heterogeneous population consisting of impulsive, proportional and radical agents the initial oscillations are smoothed and, when the new link is added, most but not all of the agents use it (Figure 6). When considering a single run it is possible to obtain a dynamics similar to the one we observed with the human participants (Figure 2). In fact, we have no oscillations for the first two turns (as with proportional agents); then some oscillations occurs (as with impulsive agents). When the new link is added most agents switch to the link with a minority following the old routes (radical agents). In this sense, the population hetereogeneity we found analyzing the qualitative data is confirmed by the simulation results. The simulation allows us to actually replicate a mixture of player types whose existence was suggested in Rapoport et al. (2009). On the contrary, from the comparison of the average of the repeated simulations we might be induced to attribute that the fraction of agents playing either SLE or SRE is due to the presence of proportional agents only and not to radical ones; nevertheless we included radical agents because this behavior emerged from the analysis of the qualitative data obtained from the participants.
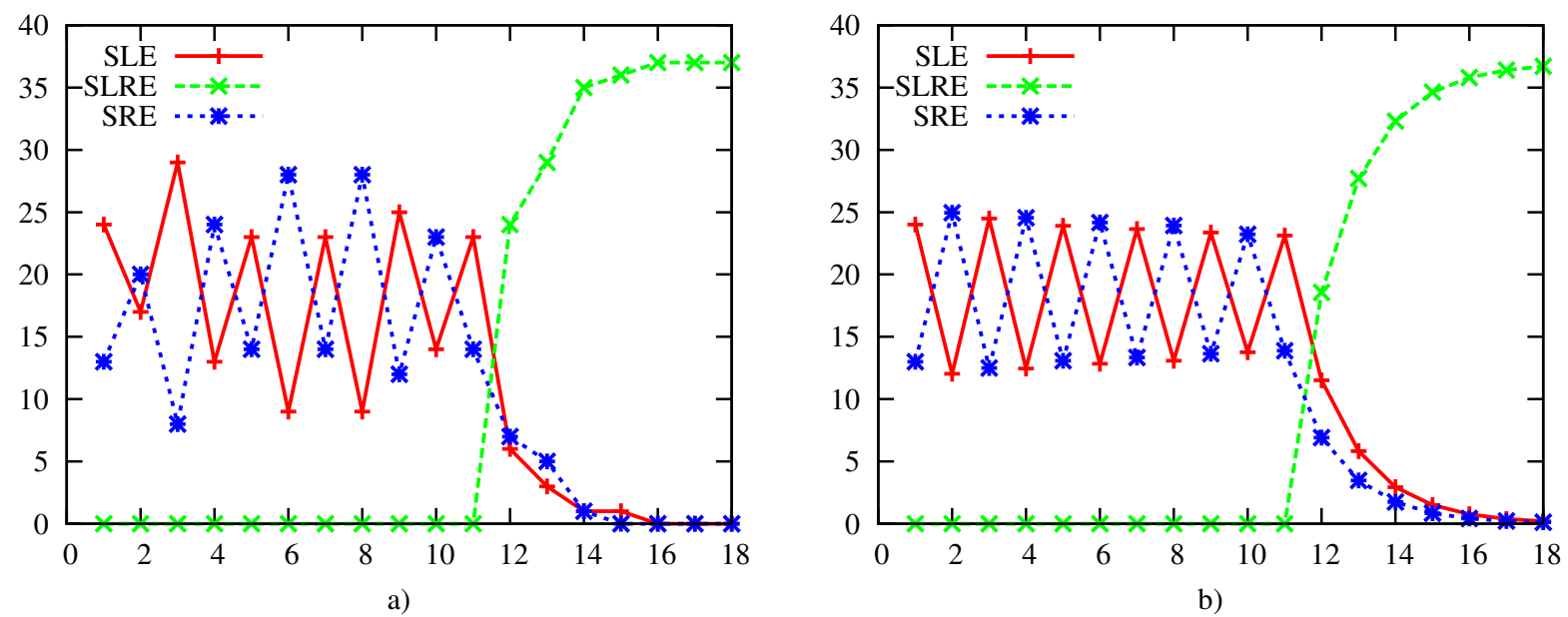

Figure 3: Homogeneous impulsive population with 37 agents. At the first turn 24 play SLE and 13 play SLR. The new link is added at turn $11:$ a) one single run; b) average of 1000 runs.

\section{CONCLUSION AND FURTHER RESEARCH}

The main goal of this paper was to establish conditions about the composition of the artificial population, in order to replicate the observed behavior of the human participants to an experiment based on the Braess paradox. In order to achieve this goal we proceeded through three steps. The first step was to run the experiment with human participants. On this occasion we gathered both quantitative and qualitative data. The quantitative data provided knowledge about the use of the different roads available. With no surprise, 


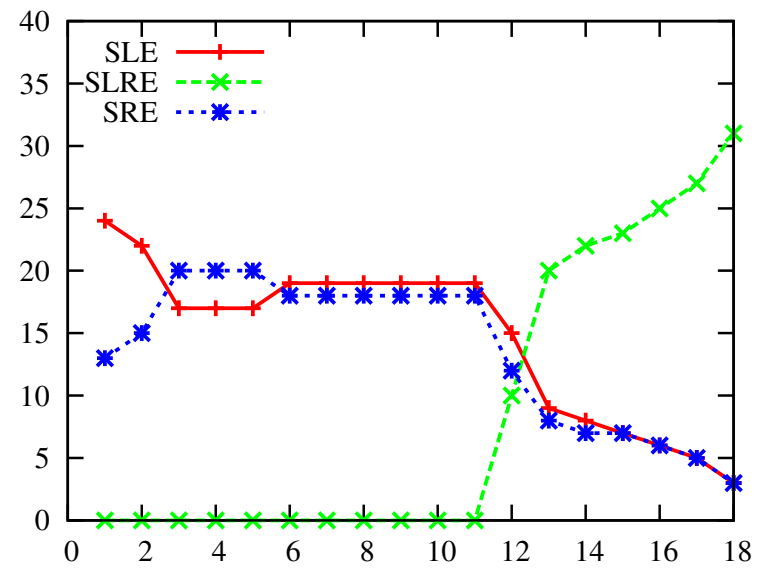

a)

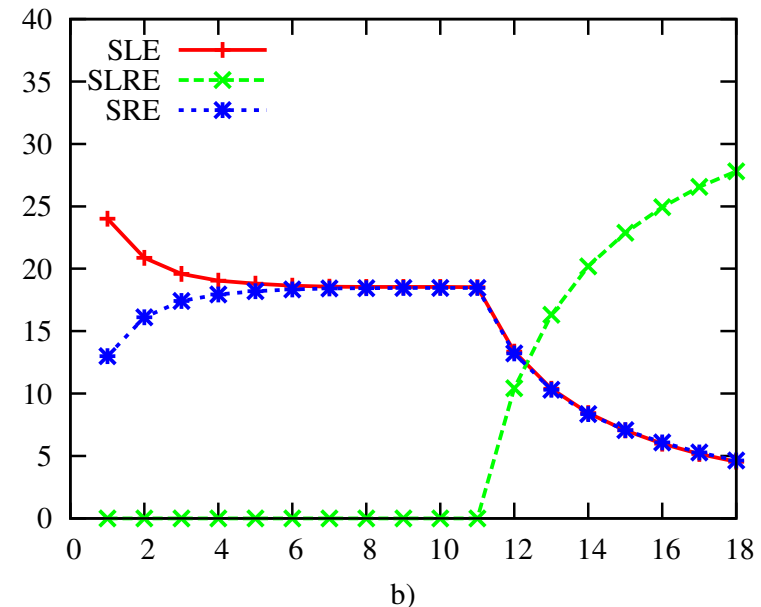

b)

Figure 4: Homogeneous proportional population with 37 agents. At the first turn 24 play SLE and 13 play SLR. The new link is added at turn $11:$ a) one single run; b) average of 1000 runs.

the paradox was confirmed: the individual rationality led to the inevitable collective irrationality. The qualitative data confirmed the existence of impulsive behavior but also provided evidence for a different behavior which was also considered in our analysis. The second step consisted in analyzing the collected data to model different behaviors for the artificial agents. The behaviors that emerged were mainly two: one (impulsive) prone to change strategy impulsively after an even small preference of a road over the others; the other one (radical) firmly standing on a selected strategy even if strictly dominated by others. The proportional behavior did not emerged from the data gathered during the experiment. The simulation with this kind of population does not reflect any features of the human population, suggesting that the impulsive behavior may be more realistic. The third and last step was to find what behavior for artificial agents was able to replicate the human experiment dynamics. Surprisingly, no homogeneous populations gave the expected result. A dynamics similar to what we observed with human participants could be obtained only with heterogeneous population, that is, with a mix of impulsive and radical behaviors. This confirms the hypothesis of a mixture of player types as suggested in Rapoport et al. (2009).

In further research we will analyze the dynamics we found with simulations in order to provide an analytical description of the dynamics depending on the relative composition of the population.

\section{REFERENCES}

Axelrod, R. 1984. The Evolution of Cooperation. New York, NY: Basic Books.

Bischi, G. I., L. Gardini, and U. Merlone. 2009a. "Impulsivity in Binary Choices and the Emergence of Periodicity". Discrete Dynamics in Nature and Society Volume 2009:Article ID 407913, 22 pages doi:10.1155/2009/407913.

Bischi, G. I., L. Gardini, and U. Merlone. 2009b. "Periodic Cycles and Bifurcation Curves for OneDimensional Maps with Two Discontinuities". Journal of Dynamical Systems \& Geometric Theories 7 (2): 101-123.

Bischi, G. I., and U. Merlone. 2009. "Global Dynamics in Binary Choice Models with Social Influence". The Journal of Mathematical Sociology 33 (4): 277-302.

Boyce, D. E., H. S. Mahmassani, and A. Nagurney. 2005. "A Retrospective on Beckmann, McGuire and Winstens Studies in the Economics of Transportation". Papers Regional Sciences 84:85-103.

Braess, D. 1968. "Über ein Paradoxon der Verkehrsplanung". Unternehmensforschung 12:258-268.

Dal Forno, A., L. Gardini, and U. Merlone. 2012. "Ternary Choices in Repeated Games and Border Collision Bifurcations". Chaos Solitons and Fractals 45 (3): 294-305. 


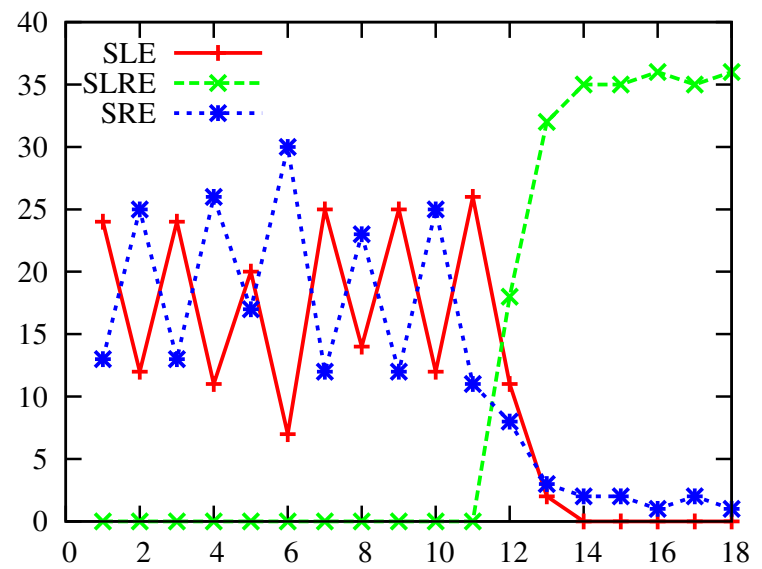

a)

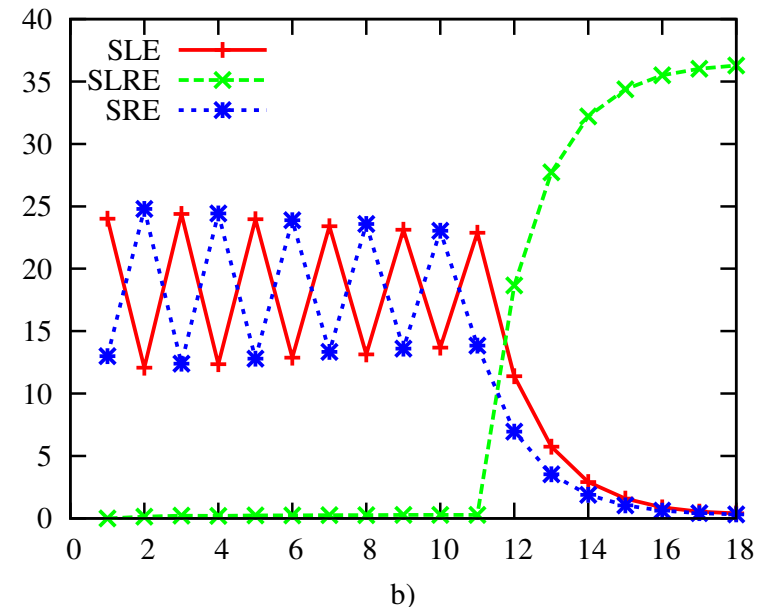

b)

Figure 5: Homogeneous radical population with 37 agents. At the first turn 24 play SLE and 13 play SLR. The new link is added at turn $11:$ a) one single run; b) average of 1000 runs.

Dal Forno, A., V. Giorgino, and U. Merlone. 2013. "Agent-Based Modeling with GT Methodology. An Example". Working Paper.

Dal Forno, A., and U. Merlone. 2012. "Grounded Theory Based Agents". In Proceedings of the 2012 Winter Simulation Conference, edited by C. Laroque, J. Himmelspach, R. Pasupathy, O. Rose, and A. M. Uhrmacher. Piscataway, New Jersey: Institute of Electrical and Electronics Engineers, Inc.

Dal Forno, A., and U. Merlone. 2013. "Border-Collision Bifurcations in a Model of Braess Paradox". Mathematics and Computers in Simulation 87:1-18.

Dawes, R., and D. M. Messick. 2000. "Social Dilemmas". International Journal of Psychology 35 (2): $111-116$.

Gardini, L., U. Merlone, and F. Tramontana. 2011. "Inertia in Binary Choices: Continuity Breaking and Big-Bang Bifurcation Points". Journal of Economic Behavior \& Organization 80:153-167.

Gisches, E., and A. Rapoport. 2012. "Degrading Network Capacity May Improve Performance: Private Versus Public Monitoring in the Braess Paradox". Theory Decision 73:267-293.

Glaser, B., and A. L. Strauss. 1967. The Discovery of Grounded Theory: Strategies for Qualitative Research. Hawthorne NY: Aldine de Gruyter.

Hauert, C., and G. Szabo. 2003. "Prisoner's Dilemma and Public Goods Games in Different Geometries: Compulsory Versus Voluntary Interactions". Complexity 8:31-38.

Janert, P. K. 2010. Gnuplot in Action. Understanding Data with Graphs. Greenwick, CT: Manning Publications Co.

Loftus, G. R. 1993. "Visual Data Representation and Hypothesis Testing in the Microcomputer Age". Behavior Research Methods, Instrumentation, \& Computers 25:250-256.

Merlone, U., D. R. Sandbank, and F. Szidarovszky. 2012. "Systematic Approach to N-Person Social Dilemma Games: Classification and Analysis". International Game Theory Review 14 (3): 1-25.

Merlone, U., D. R. Sandbank, and F. Szidarovszky. 2013. "Equilibria Analysis in Social Dilemma Games with Skinnerian Agents". Mind \& Society 18 (2): 205-217.

Morgan, J., H. Orzen, and M. Sefton. 2009. "Network Architecture and Traffic Flows: Experiments on the PigouKnightDowns and Braess Paradoxes". Games and Economic Behavior 66:348-372.

Rapoport, A., T. Kugler, S. Dugar, and E. J. Gisches. 2008. "Braess Paradox in the Laboratory: An Experimental Study of Route Choice in Traffic Networks with Asymmetric Costs". In Decision Modeling and Behavior in Uncertain and Complex Environment, edited by T. Kugler, J. C. Smith, T. Connolly, and Y. J. Son, 309-337. New York, NY: Springer. 


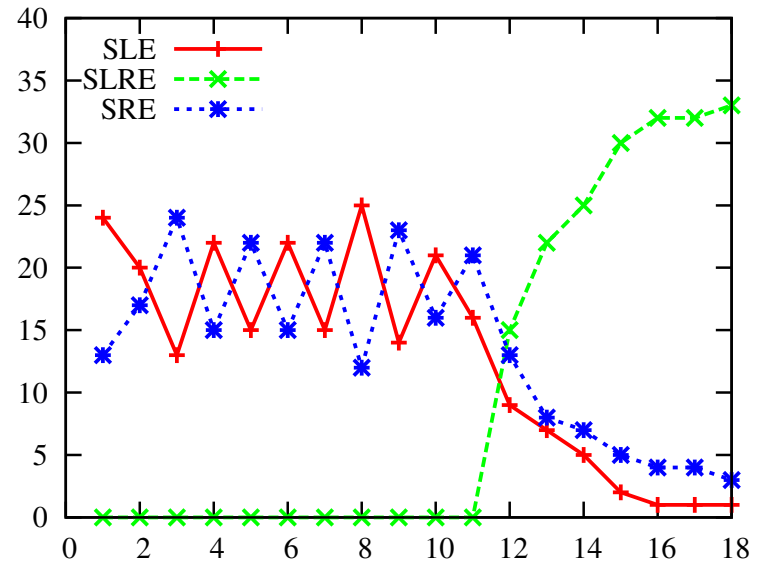

a)

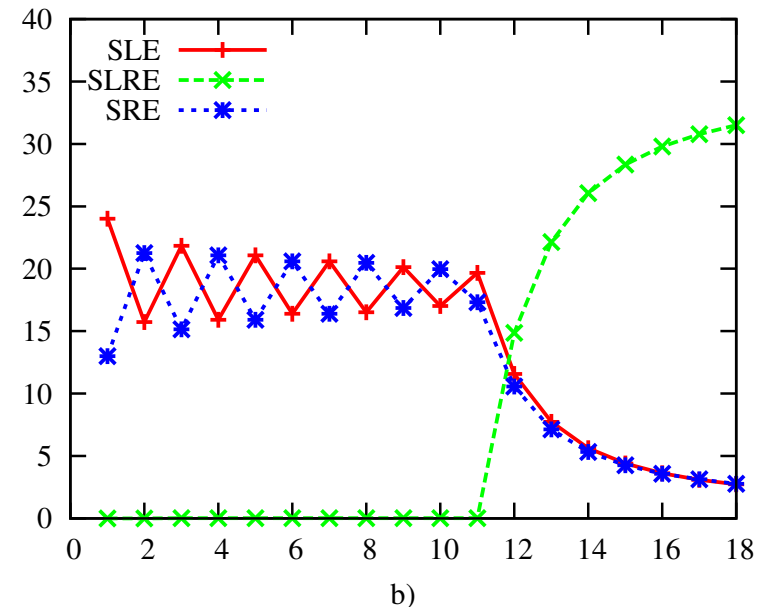

b)

Figure 6: Heterogeneous population with 37 agents: 18 impulsives, 17 proportionals, 2 radicals. At the first turn 24 play $S L E$ and 13 play $S L R$. The new link is added at turn $11:$ a) one single run; b) average of 1000 runs.

Rapoport, A., T. Kugler, S. Dugar, and E. J. Gisches. 2009. "Choice of Routes in Congested Traffic Networks: Experimental Tests of the Braess Paradox”. Games and Economic Behavior 65:538-571.

Rapoport, A., V. Mak, and R. Zwick. 2006. "Navigating Congested Networks with Variable Demand: Experimental Evidence". Journal of Economic Psychology 27:648-666.

Santos, F. C., J. M. Pacheco, and T. Lenaerts. 2006. "Evolutionary Dynamics of Social Dilemmas in Structured Heterogeneous Populations". Proceedings of the National Academy of Sciences of the United States of America 103 (9): 3490-3494.

Smith, M. J. 1979. "The Existence, Uniqueness, and Stability of Traffic Equilibria". Transportation Research 13B:295-304.

Steinberg, R., and W. I. Zangwill. 1983, August. "The Prevalence of Braess' Paradox". Transportation Science 17 (3): 301-318.

Strauss, A. L., and J. M. Corbin. 1998. Basics of Qualitative Research: Techniques and Procedures for Developing Ground Theory. 2nd ed. Thousand Oaks CA: SAGE Publication, Inc.

\section{AUTHOR BIOGRAPHIES}

ARIANNA DAL FORNO holds a degree in Mathematics and a Doctorate in Mathematics applied to Economic Decisions. She has been visiting Professor at the Universidad de Chile, post-doc at the Department of Economics at Harvard University. She is author of several papers published in international journals as Chaos, Solitons and Fractals, European Journal of Operational Research, Journal of Economic Behavior \& Organization, Nonlinear Dynamic, Psychology and Life Sciences, Mathematics and Computers in Simulation. She is also referee for JASSS, Decisions in Economics and Finance and Communications in Nonlinear Science and Numerical Simulation. Her email address is arianna.dalforno@unito.it.

UGO MERLONE (Ph.D. in Applied Mathematics, University of Trieste, Italy) is Associate Professor at the Psychology Department, University of Torino, Italy. His main area of interest is the modeling of human behavior and organizations. On these topics he has published on journals such as European Journal of Operational Research, Physica A, Journal of Economic Behavior \& Organization, Journal of Mathematical Sociology, International Game Theory Review, Organization Science, Journal of Artificial Societies and Social Simulation, Mathematics and Computers in Simulation, and Communications in Nonlinear Science 


\section{Dal Forno and Merlone}

and Numerical Simulation. Further details can be found on his homepage www.ugomerlone.net. His email address is ugo.merlone@unito.it. 